\title{
La création des Écoles supérieures du professorat et de l'éducation s'inscrit-elle dans un modèle supranational de professionnalisation des enseignants?
}

Guy Lapostolle

\section{OpenEdition}

\section{Journals}

Édition électronique

URL : http://journals.openedition.org/trema/3069

DOI : $10.4000 /$ trema.3069

ISSN : 2107-0997

Éditeur

Faculté d'Éducation de l'université de Montpellier

Édition imprimée

Date de publication : 1 décembre 2013

Pagination : $60-75$

ISSN : 1167-315X

Référence électronique

Guy Lapostolle, «La création des Écoles supérieures du professorat et de l'éducation s'inscrit-elle dans un modèle supranational de professionnalisation des enseignants ? », Tréma [En ligne], 40 | 2013, mis en ligne le 01 décembre 2015, consulté le 02 mai 2019. URL : http://journals.openedition.org/ trema/3069 ; DOI : 10.4000/trema.3069

Ce document a été généré automatiquement le 2 mai 2019.

Trema 


\title{
La création des Écoles supérieures du professorat et de l'éducation s'inscrit-elle dans un modèle supranational de professionnalisation des enseignants?
}

\author{
Guy Lapostolle
}

1 À partir des années 1990, la formation des enseignants va connaître dans de nombreux pays de profonds bouleversements. Un mouvement de "professionnalisation", qui trouve ses origines aux États-Unis, dans les années 1980 va s'imposer progressivement aux États dans lesquels les politiques de massification engagées dans les années 1950, semblent marquer le pas (Tardif, 2010).

2 Pour autant, ce mouvement n'a pas donné lieu à une uniformisation de la formation des enseignants dans tous ces États. Un processus de «créolisation ", selon l'expression d'Anderson-Levitt (2003) a été à l'œuvre, qui conduisait les acteurs politiques, mais aussi les acteurs opérationnels, à interpréter, en fonction de

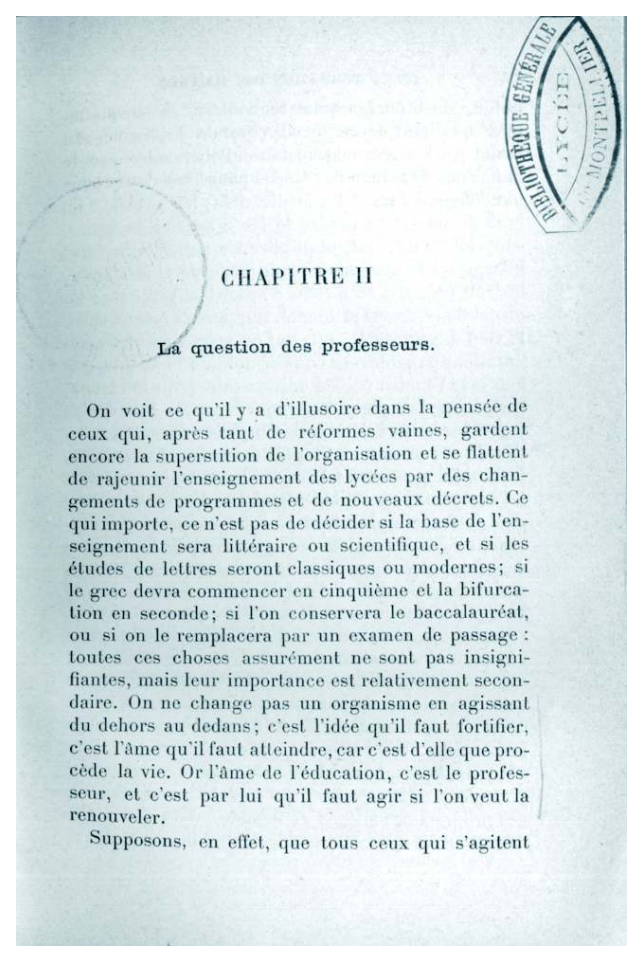


leur histoire et de leurs particularités, le modèle et les recommandations supranationales. La France n'a pas échappé à ce processus de " créolisation ». Une des manières dont on a adapté le modèle dans les IUFM (Instituts universitaires de formation des maitres), jusqu'à la réforme dite de masterisation, a conduit à porter l'attention sur la posture des enseignants en formation. Les textes cadrant au niveau national cette formation en France témoignent d'un souci très présent de ce qu'il est convenu d'appeler un processus de "déscolarisation» qui accompagne la professionnalisation des enseignants en formation. Or il semble que ni la réforme de la masterisation au tournant des années 2009-2010, ni la création des ESPE, à la rentrée 2013, n'ont intégré cette expérience acquise au sein des IUFM. Tout semble s'être passé comme si la volonté de faire converger la formation vers un modèle supranational, dont on ne retenait que les grandes tendances, tout en prétendant "faire du neuf», avait conduit les acteurs politiques à « faire table rase » de l'expérience acquise.

3 L'objet de cet article est d'apporter, en proposant une mise en perspective historique, quelque éclairage quant à la manière dont la France s'est inscrite dans ce mouvement supranational. Il est également de montrer les incidences de ces choix et de ces traductions sur la formation des enseignants. Les effets des choix opérés et de leurs mises en œuvre seront alors appréhendés à l'aune de la manière dont les enseignants en formation s'approprient cette formation. Davantage que les contenus de formation prescrits, c'est cette manière dont les étudiants et stagiaires s'approprient ces contenus qui permettra d'envisager les effets des réformes de la formation des enseignants du début des années 1990 à nos jours.

Dans un premier temps, nous montrerons que le modèle supranational, s'il semble avoir influencé la formation des enseignants dans de nombreux États, n'en présente pas moins des contours mouvants qui lui ont permis de s'imposer en dépit des diverses critiques qu'il rencontrait. Dans un deuxième temps, nous montrerons comment il a été mis en œuvre en France dans les IUFM et comment la masterisation, considérée comme une des évolutions à laquelle invitait le modèle, a contribué à modifier l'organisation de la formation. Dans un troisième temps, nous montrerons que cette masterisation a porté atteinte au processus fondamental de professionnalisation/déscolarisation qui sous-tend une formation des enseignants exigeante. Enfin, dans un quatrième temps, nous tenterons d'observer, compte tenu des quelques indicateurs dont nous disposons actuellement, si les ESPE sont en mesure de reprendre à leur compte ce processus au fondement de bon nombre de pratiques de formation, corroborées par quelques vingt années d'expérience, mais aussi par de nombreux retours réflexifs et scientifiques sur ces expériences.

\section{De l'accueil et des traductions d'un mouvement de «professionnalisation »}

5 Un certain nombre de recherches européennes rendent compte de la manière dont ce modèle supranational de "professionnalisation» a été accueilli et «traduit» dans les différents Etats qui se sont inscrits dans ce mouvement.

Mellouki et Wentzel (2009)1 ${ }^{1}$, étudiant les usages de ce modèle en Suisse romande, définissent en fait cette "professionnalisation» comme une "norme internationale 
flottante ». Celle-ci se caractérise par des tendances qui influencent les systèmes de formation des Etats d'Europe. Ces tendances sont les suivantes :

- Tertiarisation ou universitarisation de la formation;

- Prise en compte des résultats de la recherche en éducation ;

- Hausse du temps réservé à la formation pratique ;

- Valorisation de la formation continue et son insertion dans le processus d'apprentissage tout au long de la carrière ;

- Encouragement d'approches de formation et d'enseignement tournées vers la réflexion critique des apprenants et des praticiens;

- Adoption d'un référentiel de compétences.

7 Pour autant, selon les auteurs, le modèle peine à être intégré dans les programmes d'action qui ciblent la formation des enseignants. Ils mentionnent qu'en 2006, la Commission suisse de la recherche en éducation (CSRE) observait que "jusqu'ici, aucun consensus n' [avait] été trouvé autour du sens à donner à cet objectif et de la manière de l'atteindre ». Si tel était le cas, c'est que "la légitimité scientifique des standards [restait] controversée " et que les "modèles idéaux" de professionnalisation qui ont inspiré les réformes étaient "impossibles à réaliser dans l'exercice de la profession». Les auteurs avancent quelques explications quant aux dysfonctionnements engendrés lors de la mise en œuvre du modèle : «En 2007, Bagnoud relève pour sa part l'amalgame de modèles dans les nouvelles politiques de formation des enseignants. Pendant que l'organisation scolaire fonctionne encore selon les anciennes conceptions et les normes bureaucratiques, les nouveaux programmes de formation s'inspirent, quant à eux, du modèle professionnel, aux contours imprécis. Ce flottement génère d'étranges configurations, qui empruntent aux uns et aux autres modèles certains aspects par définition incompatibles ou contradictoires, engendrant de fait, pour les acteurs, une difficile adaptation à une nouvelle, mais surtout insaisissable, culture professionnelle ».

8 Maulini (2008), citant le Grand Conseil de la République et du Canton de Genève, à propos d'un projet de loi, montre comment il ne va pas de soi de s'inscrire dans ces grandes orientations supranationales : "À l'évidence la formation d'un instituteur justifie une formation théorique de 189 crédits complétée par un stage pratique au front des classes. Il serait toutefois absurde d'exiger, pour l'activité visée d'un instituteur, un master, soit 300 crédits, nécessitant dans la règle près de 5 ans d'études [...] Sans même évoquer d'éventuelles conséquences financières pour l'université et pour l'État employeur et pour les instituteurs, on ne peut envisager de dilater la formation d'un instituteur à l'égal d'un avocat, d'un architecte, voire d'un médecin. L'expérience pratique d'un instituteur demeure incomparable, si bonne soit sa formation théorique » (PL 9500, 2005). L'auteur montre comment quelques années plus tard alors que la réforme masterisation a eu lieu, les réactions au sein du Grand Conseil témoignent toujours de positions d'acteurs divergentes sur l'intégration du modèle supranational : «À Genève, les candidats à l'enseignement doivent suivre une formation de cinq ans avant de pouvoir prétendre à un diplôme qui leur permettra d'enseigner. C'est trop long. Le résultat de cette politique est que nous formons, au sein de la Faculté, des enseignants qui ont dû acquérir un vaste savoir théorique sur cinq ans, mais qui ont été moins que dans d'autres cantons confrontés à des situations pratiques. De plus, l'enseignement prodigué à la Faculté de psychologie et de sciences de l'éducation, quelle que soit sa qualité, se revendique comme un enseignement universitaire qui, au besoin, doit pouvoir se détacher des contingences professionnelles et des obligations de l'Étatemployeur. C'est pourquoi si la Faculté a tout son sens notamment dans la liberté académique, elle est en revanche moins adaptée à la mission que souhaite lui confier l'État, à savoir la formation pratique du personnel enseignant. À plus forte raison quand on sait que la quasi-totalité des cadres 
de cette Faculté se revendiquent de choix pédagogiques en opposition frontale avec ceux plébiscités par le peuple genevoix lors de la votation de 2006 et confirmés quelques mois plus tard par le règlement d'application ». (PL10316, 2008).

D'autres exemples de tentative de mise en conformité avec ce modèle pourraient être cités (Baillat, Niclot, Ulma, 2010). Ils confirmeraient cette difficulté que chaque État rencontre lorsqu'il cherche à épouser le modèle. Son histoire, le contexte dans lequel il adopte, plus ou moins activement, le modèle sont autant de facteurs qui déterminent la manière dont les acteurs politiques ou opérationnels acceptent ou résistent à cette adoption. Comme le mentionne Tardif (2010) : «Il y a fort à parier que cela prendra plusieurs décennies avant d'aboutir à une véritable harmonisation, surtout dans le domaine de la formation des enseignants [...] une telle harmonisation reste superficielle et ne touche pas ou peu les idéologies, les institutions et les pratiques de formation dans chaque pays ».

Ainsi chaque État tend à réinterpréter le modèle supranational dans le contexte national qui est le sien. Il convient d'observer la manière dont la France a, elle aussi, donné une forme particulière à ce mouvement de professionnalisation dans lequel elle entendait s'inscrire.

\section{De la création des IUFM à la masterisation}

11 Plusieurs réformes vont se succéder du début des années 1990 à $2010:$ la mise en place des IUFM au début des années 1990, l'intégration des IUFM dans l'université en 2005, la formation des étudiants au niveau du master et la réforme des concours de recrutement des enseignants, en 2010.

Au début des années 1990, la création des IUFM (Instituts universitaires de formation des maîtres) témoigne de la volonté de prendre en compte les évolutions d'une professionnalité enseignante qui se voit soumise à de nouvelles exigences. Ces exigences sont liées en partie au processus de démocratisation de l'enseignement secondaire dont on peut dire qu'il s'accélère depuis la seconde partie du XXe siècle. Ce processus a en effet transformé une école primaire qui n'est plus sa propre fin mais qui prépare l'entrée de quasiment tous les élèves au collège, puis au lycée. L'enseignement secondaire voit les flux d'élèves qu'il accueille augmenter de manière significative. À titre d'illustration, la proportion de bacheliers sur une génération est passée de $3 \%$ en 1945, à $25 \%$ en 1975 , pour atteindre 71,6\% en 2011.

13 Cette «nouvelle » formation participe aussi d'un mouvement européen, voire mondial, qui invite à adosser la formation des enseignants du premier degré, mais aussi du second degré, à l'université. La France pouvait-elle encore continuer de former les premiers dans des Écoles normales en marge de l'université alors que la Grèce, l'Espagne et le Portugal, des «puissances moyennes » selon l'expression de Robert et Terral (2000), proposaient pour ces enseignants des formations universitaires? De même, la formation des enseignants du second degré, après leur réussite au concours, dans des CPR (centres pédagogiques régionaux) sous l'autorité des inspecteurs, pouvaient-elle demeurer à l'écart de l'université alors que dans de nombreux États, cette formation incombait aussi aux départements de pédagogie des universités, en lien étroit avec des établissements scolaires.

14 Avec les IUFM, se mettent alors en place un recrutement et une formation des enseignants qui conserveront une certaine stabilité jusqu'en 2010. Les étudiants doivent 
être titulaires d'une licence pour prétendre passer le concours de recrutement. Cette licence obtenue, ils se préparent pendant un an au concours au sein des IUFM. Une fois le concours obtenu, ils sont «fonctionnaires stagiaires». Ils sont en «stage en responsabilité » devant des élèves, dans des établissements à temps partiel, à raison de 6 à 8 heures/semaines pour le second degré, et en stage filé sur une journée/semaine, auquel s'ajoutent deux stages massés, pour le premier degré, pendant un an, tout en suivant une formation au sein des IUFM. A la fin de cette année, ils sont ou non titularisés à la suite d'une inspection effectuée par un inspecteur, membre de l'Éducation nationale.

Cette organisation connaît un bouleversement relativement médiatisé au tournant des années 2010, sous l'effet de la réforme de la "masterisation». Celle-ci est une des conséquences du processus de Bologne, initié en1999, qui a conduit à la construction de l'Espace européen de l'enseignement supérieur, et qui invite en effet la France à élever le niveau de la formation des enseignants. Mais cette «masterisation " s'inscrit aussi dans une volonté d'universitariser une formation conformément au modèle de professionnalisation dont Mellouki et Wentzel (op.cit.) rappelaient les grandes tendances : « tertiarisation ou universitarisation » de la formation et prise en compte des résultats de la recherche en éducation dans cette formation. Dans les faits, cette «masterisation » est opérationnalisée par trois réformes.

La première de ces réformes concerne l'intégration des IUFM dans l'université (Ministère de l'éducation nationale, 2005). Elle a son corollaire : la responsabilité de la formation des « fonctionnaires stagiaires » revient en grande partie à l'État employeur, au ministère de l'Éducation nationale, alors qu'auparavant elle était davantage partagée entre les IUFM et l'État employeur. L'intégration des IUFM se manifeste principalement par le fait que ceux-ci sont désormais dépendants des décisions prises par le conseil d'administration des universités dans lesquels ils sont intégrés. Ils ne sont plus comme auparavant des établissements jouissant d'une relative autonomie, avec leur propre conseil d'administration, présidé par le Recteur d'académie, représentant du ministre de l'Éducation nationale. Ils sont sous l'autorité du président d'université. L'intégration des IUFM dans l'université entérine donc la responsabilité de l'université dans la formation des enseignants en devenir, lorsqu'ils sont encore étudiants. Dans le même temps, la formation des « fonctionnaires stagiaires » est placée sous une plus stricte responsabilité de l'Etat employeur.

La seconde réforme modifie le niveau de recrutement des enseignants et la troisième les modalités de leur formation. Ces réformes conduisent à recruter les enseignants au niveau du master et selon un concours nouveau passé pendant la seconde année de master. Elles modifient profondément l'organisation qui prévalait depuis 1990 dont les principales caractéristiques étaient les suivantes :

- La préparation au concours se faisait à temps complet puisqu'elle était déconnectée de l'obtention du diplôme. La licence étant déjà acquise, la première année à l'IUFM était exclusivement consacrée à la préparation du concours.

- Lors de la seconde année, la formation des stagiaires s'effectuait en alternance avec une partie du temps passé en établissement en responsabilité devant des élèves, accompagnés dans cette tâche par un collègue, appelé tuteur mais aussi, bien souvent, par des formateurs IUFM, et l'autre partie de ce temps en IUFM pour apporter, entre autres, des éléments permettant d'analyse les pratiques mises à l'épreuve lors de ce stage.

L'effet immédiat de ces réformes est que les candidats préparent un concours en même temps que le diplôme de master. Or le fait que les épreuves du concours ont lieu au début 
de la seconde année de master pour les épreuves écrites et à la fin de cette seconde année pour les épreuves orales oblige à organiser de manière particulière les contenus d'enseignement du master, notamment en seconde année. Compte tenu de l'importance $\mathrm{du}$ concours pour les jeunes étudiants, les masters qui traditionnellement ont pour objectif de former les jeunes étudiants à la recherche et/ou de les professionnaliser, doivent voir leurs contenus organisés de sorte à permettre aux étudiants de réussir aux concours.

19 Ces organisations différentes de la formation des enseignants ont alors des implications non négligeables sur les dispositifs et contenus de formations. Elles conduisent aussi à des différences fondamentales quant aux modalités d'évaluation mises en œuvre dans le cadre de cette formation initiale.

\section{Des conséquences sur le processus de déscolarisation}

20 Du début des années 1990 à 2010, les deux années de formation en IUFM étaient distinctes. En première année, les étudiants préparaient un concours, ils étaient en période de "bachotage », même si quelques stages «accompagnés » leur permettaient, lorsqu'ils n'avaient pas bénéficié d'une "pré professionnalisation » dans leur cursus de licence, un premier contact avec des élèves. En seconde année, en revanche, ils étaient devenus "professeurs stagiaires». Ils étaient de six à huit heures en responsabilité devant des élèves et poursuivaient une formation parallèle dans les IUFM.

De ce fait, l'évaluation de ces enseignants en formation était clairement identifiable. En première année, le concours servait de juge de paix. Cette modalité d'évaluation, que l'on peut qualifier de "scolaire", notamment en raison des épreuves encore très académiques, ne traduisait pas l'acquisition de compétences strictement professionnelles. Pour autant, les étudiants étaient dans une posture claire par rapport à ce que l'on attendait d'eux, ils avaient un objectif, celui de réussir le concours. La seconde année, quant à elle, conduisait à des modalités d'évaluation plus en rapport avec l'exercice du métier. Les IUFM validaient la formation des stagiaires sur la base de modules, d'un mémoire professionnel et d'un stage qui constituait le terrain d'étude du mémoire et qui était évalué par les formateurs d'IUFM. Si les modules et le mémoire concouraient à l'élaboration du dossier communiqué à l'Etat employeur, dans la procédure de "validation", le stage restait prépondérant dans cette procédure de validation de la formation des jeunes enseignants (Lapostolle, Maurel, Verney-Carron, 2007). Quelques changements néanmoins allaient néanmoins apparaître avant la masterisation.

À partir de 2007, les IUFM sont tenus de respecter un cahier des charges de la formation des enseignants (MEN, 2007). Si la formation en première année est toujours préparatoire aux concours de recrutement, la formation en deuxième année, pour les fonctionnaires stagiaires est modifiée. Les IUFM ne valident plus cette seconde année. L'examen de qualification professionnelle - à partir duquel sera décidée ou non la titularisation des stagiaires - se fera sans l'appui du rapport rendu par les IUFM. La commission chargée, au nom de l'État employeur, de la titularisation des stagiaires, se passera de l'avis rendu par les IUFM.

Dans le même temps que le cahier des charges formulé en termes de compétences devient le guide de la mission des IUFM, le mémoire professionnel n'est plus mentionné comme 
une épreuve obligatoire, aucune consigne n'étant donnée quant aux modules auparavant évalués à des fins de validation de la formation. Quant aux stages, s'ils sont encore encadrés par des formateurs d'IUFM, ces derniers ne sont pas consultés en vue de la titularisation des stagiaires.

En somme, cette moindre importance accordée à quelques-unes des dimensions de la formation portées par les IUFM, de même le cadrage de leur mission non plus en terme de contenus (formation générale et transversale, stages...) mais en termes de "compétences", peut se comprendre comme un recentrage de la formation sur les réalités de l'exercice du métier ${ }^{2}$. On peut noter à ce propos que le système français se rapproche du système anglais de formation des enseignants. Mallet, Brisard (2003) remarquent que les critiques adressées à la formation théorique en Angleterre, dans divers rapports publiés à partir des années 1970, ont contribué à ce que cette formation théorique et les sciences humaines et sociales qui la sous-tendaient cèdent progressivement la place à des thèmes plus concrets, visant le développement de compétences professionnelles.

La masterisation perturbe alors ou conduit à une certaine confusion quant à ce recentrage sur les compétences. Notamment parce que les modalités et la nature des évaluations vont être bouleversées. Le fait que les contenus d'enseignement sont organisés et évalués selon les règles exigibles pour les masters modifie le rapport que les étudiants ont à ces contenus. Le simple fait d'une évaluation par des notes modifie le rapport des étudiants aux contenus de formation proposés. Dans une certaine mesure, cette évaluation redonne une certaine légitimité à des contenus qui ne trouvaient pas toujours un grand intérêt auprès des étudiants dans le cadre des modalités de formation antérieures. "La formation générale et transversale $»^{3}$, notamment, peinait à être légitime aux yeux des étudiants puisqu'elle n'était d'aucune utilité pour la préparation aux concours et qu'en seconde année de formation, les stagiaires dans l'urgence de la préparation de leurs cours, restaient davantage intéressés par des savoir-faire dont ils pouvaient faire un usage immédiat (Lapostolle, Mabilon-Bonfils, Genelot, 2009). Désormais prise en compte dans l'évaluation qui conduit à l'obtention du diplôme, les étudiants trouvent par nécessité, un intérêt à cette formation.

Ceci étant, le fait que, lors des deux années de master, tous les contenus de formation sont désormais notés - des contenus les plus théoriques aux plus pratiques comme les stages -, donne naissance à un paradoxe. Alors que la professionnalisation est envisagée comme un "processus de déscolarisation »- Pour résumer la tentative de définition du terme par Abraham (2007), on peut dire que l'étudiant va progressivement cesser de se soucier de la note pour chercher à gagner en efficacité sur le terrain, pour devenir plus " compétent »-, les évaluations proposées dans le cadre du master tendent à renforcer la "forme scolaire» des contenus de formation. Saussez et Allal (2007) diraient que ces modalités d'évaluation incitent les étudiants à "se faire paon ", pour se montrer sous leur meilleur jour à leur formateur, ou encore caméléon, pour se conformer à ses attentes. Ces évaluations confortent une attitude des étudiants que l'on pourrait qualifier de « scolaire ", d'autant que la préparation aux concours, prioritaire aux yeux des étudiants, existe encore à côté de cette nouvelle logique diplômante.

En somme, au cours de ces deux années de master, les exigences du concours et du diplôme confortent une posture des étudiants peu propice à une réelle professionnalisation. C'est seulement lorsque les étudiants deviennent stagiaires, lorsqu'ils sont libérés du concours et du diplôme que l'exercice réel du métier devient la 
référence à partir de laquelle ils sont évalués et qu'ils peuvent se consacrer pleinement à l'apprentissage de ce métier. En fait, les causes des dysfonctionnements occasionnées par cette "masterisation" ne sont pas imputables à la masterisation en soi, ni à une prolongation du statut d'étudiant, ni à une survalorisation de la théorie par rapport à la pratique, comme on a pu le lire dans de nombreux rapports français ou étrangers. Ces causes semblent davantage liées au fait que l'on ne sache pas prendre réellement en compte les transformations des postures des ceux qui se destinent au métier d'enseignant au cours du processus de leur formation.

De nombreuses analyses, françaises ou étrangères s'arrêtent bien souvent à ces grands constats ou à ces grandes dénonciations qui présentent l'avantage d'être facilement compréhensibles pour - et admises par - l'opinion publique, y compris celle des enseignants en formation eux-mêmes. Cependant, ces constats évincent cette question des postures.

Dans le cas du canton suisse cité précédemment, les causes des dysfonctionnements occasionnés par la masterisation sont liées principalement à la survalorisation de la théorie et à un manque de confrontation des étudiants à la réalité du métier. On peut à cet égard remarquer que cette analyse est du même ordre que celle effectuée par les Anglais dans les années 1970 (Mallet, Brisard, op.cit.), ou encore que celle du ministre Darcos (2009) qui évoquait, entre autres, une survalorisation de la théorie ${ }^{4}$ dans la formation des enseignants au sein des IUFM. Dans un article récent, Jamet (2013) indique qu'une tendance mondiale est de faire une place toujours plus importante aux établissements scolaires et à l'État employeur dans la formation même si par ailleurs un équilibre semble être recherché entre formation théorique et formation pratique.

Or ce qui est en jeu, de notre point de vue, tient davantage au fait de prendre (ou non) en compte les préoccupations principales et les postures des enseignants en formation. Tout autant que les contenus des formations, les dispositions dans lesquelles sont les étudiants pour se les approprier se révèlent être prioritaires.

31 Dans le cas français, depuis le début des années 1990, quelques rapports des inspections générales (IGEN, IGAEN, 2003), de même qu'un certain nombre de travaux scientifiques, avaient attiré l'attention des acteurs politiques qui semblaient jusqu'alors avoir tenu compte de ces quelques constats tirés de l'expérience des formateurs.

\section{De la prise en compte de l'expérience dans le cadre des ESPE}

32 Un ensemble de textes officiels, en apparence anodins, montrent qu'une prise de conscience quant à ce processus de « déscolarisation »- par ailleurs jamais cité comme tel - avait été encouragée par l'État, notamment à la suite d'un certain nombre de recherches et de rapports destinés à réguler le travail effectué au sein des instituts (Lapostolle, Grisoni, Solomon, 2012).

C'est par le biais de régulations qui font intervenir différents acteurs - des experts, des scientifiques, l'État, les IUFM - que la formation des enseignants s'est construite comme un processus progressif dont témoignent des modalités de formation qui s'affinent $\mathrm{du}$ début des années 1990 jusqu'à la mise en place de la masterisation. On assiste notamment à une évolution des postures des formés et des formateurs. Celles-ci sont plus en phase 
avec une réalité qui émerge au fur et à mesure que se construit l'expérience de formation et que s'éprouvent les pratiques.

Si l'on se réfère aux indicateurs que sont les positionnements des formateurs et des formés par rapport aux contenus de formation, ainsi qu'aux modalités de formation proposées dans les textes officiels, un changement notable apparaît en 2002. En 1992, les circulaires mentionnent des " élèves ", qu'il s'agisse des étudiants et des stagiaires (MEN, 1992). Si certes il faut y voir une formulation habituellement en usage dans les anciennes Ecoles normales, on ne peut s'empêcher de penser que celui qui est en formation n'a pas encore tout à fait le statut d'adulte. Il faut attendre 2002 pour que la formation des stagiaires soit définie comme une «formation d'adultes à caractère universitaire » (MEN, 2002).

Dans les faits, la circulaire de 2002 entérine le changement de posture du formé lorsqu'il passe du statut d'étudiant à celui de stagiaire. Une fois admis au concours, l'étudiant devient stagiaire et est en mesure de recevoir une formation professionnelle. À ce titre il devient " un adulte en formation », cette formation devant lui permettre d'opérer quatre mutations : "passer de la position de celui qui apprend à celui qui fait apprendre"; « accéder au statut de fonctionnaire du service public d'éducation »; « prendre en charge la dimension éducative du métier » ; « apprendre à connaître et à comprendre la diversité des contextes et des réalités scolaires».

Il est aussi prévu à la fin de la formation initiale un accompagnement des néo-titulaires (MEN, 2001). L'appropriation du métier est reconnue comme relevant d'un processus long. En fin de formation initiale, la maitrise du métier est à parfaire : «En effet, ce n'est que par une maîtrise progressive des compétences attachées à l'exercice de son métier [... ] que l'enseignant va se forger peu à peu avec le temps et au contact des réalités pédagogiques une identité professionnelle affirmée». Le fait que cette formation soit appelée d'abord «accompagnement de l'entrée dans le métier " (MEN, 2001) et ensuite «formation initiale différée » (MEN, 2007) et non plus «formation continue » montre qu'une étape supplémentaire est nécessaire pour marquer le passage de statut de stagiaire à la reconnaissance de l'enseignant confirmé. La création du terme "néotitulaire » témoigne en quelque sorte de la reconnaissance institutionnelle de la nécessité de cette étape.

Il semble alors qu'avec la création des ESPE et des nouveaux concours, ce problème de la clarification de la posture de l'enseignant en formation soit résolu, mais en partie seulement. Le fait que le concours ait lieu en première année de master libère l'étudiant lors de la seconde année de master des contraintes du "bachotage " auquel il était assujetti en raison de la préparation des oraux. Cependant, le double statut dont il fait l'objet, à la fois de « contractuel admissible » et d'« étudiant » en seconde année, fait qu'il ne pourra vraisemblablement pas être entièrement disponible pour apprendre le métier. La nécessité de réussir son diplôme de master, l'encouragera à se consacrer à des objectifs qui peuvent être en décalage avec les exigences du terrain. Pour éviter cet effet pervers, il convient que les contenus dispensés en deuxième année soient, dans une certaine mesure, susceptibles d'apporter des réponses aux problèmes rencontrés dans le cadre du stage, alors qu'il est « contractuel admissible »... Or, au lendemain de la masterisation, la tendance à vouloir maintenir un certain niveau d'exigence en matière de recherche en seconde année de master - dans des diplômes portés dans de nombreux cas par des UFR (unités de formation et de recherche), mais aussi parfois par des IUFM - tendait à mettre l'étudiant dans une perspective de travail à plus long terme. De ce fait elle a pu être 
contre productive. En tous cas, cette tendance qui enfermait l'étudiant dans des logiques et des temporalités pas toujours compatibles, ne contribuait pas à le mettre dans des dispositions favorables à une réelle appropriation des exigences du métier.

Il en était de même en ce qui concernait la multiplication des évaluations dans le cadre des masters. La nécessité de mettre des notes pour délivrer (ou non) le master a pu conduire, dans certains cas, à évaluer des enseignements de quelques heures et parfois même sous forme de QCM (Questionnaires à choix multiples). Rendre compte par écrit d'un cours de quelques heures ne garantit en rien du bon usage que l'on peut faire de ce cours dans le cadre de l'exercice d'un métier (Lapostolle, 2013).

Ainsi, quelques contenus proposés, ou certaines modalités de leur transmission/ appropriation, de même que quelques-unes des modalités de leur évaluation, plaçaient les enseignants en formation dans des postures ambiguës ou plus exactement peu propices à l'apprentissage du métier. Il restera à observer comment les ESPE sauront prendre en compte ces constats.

Il apparaît d'ores et déjà déterminant, et particulièrement pour les étudiants de seconde année de master qui seront en même temps seuls devant des élèves, lors de leur stage, que les exigences de l'État employeur, notamment celles de recruter des enseignants capables d'être rapidement opérationnels sur le terrain, et celles de l'université soient harmonisées de sorte à ne pas brouiller les postures de ces enseignants en formation. Si la masterisation avait assez strictement séparé les rôles et missions des deux institutions, la voie de la coopération entre celles-ci devient nécessaire ${ }^{5}$. Elle n'est pas une simple exigence institutionnelle, elle aura un impact majeur sur la posture des enseignants en formation, notamment sur le processus de leur professionnalisation/déscolarisation.

41 Pour autant, la professionnalisation des jeunes enseignants ne se construit pas exclusivement dans le cadre de l'exercice réel du métier. Nombreuses sont les recherches scientifiques, dans le champ de la didactique professionnelle ou encore dans les travaux relatifs à la formation des enseignants, qui montrent la nécessité de doter les enseignants d'« outils de réflexion» sur leurs pratiques. Envisagées comme des connaissances « pour » enseigner, elles sont nécessaires pour prendre de la distance par rapport aux pratiques professionnelles existantes ou connaître les conditions dans lesquelles un recours à ces pratiques est pertinent ou encore quand la culture professionnelle est impuissante pour faire face à de nouveaux problèmes... Les sciences humaines et sociales, dès lors qu'elles sont utilisées dans des conditions qui font sens pour les enseignants en formation, constituent une part non négligeable de ces outils qui permettent de les professionnaliser de manière exigeante (Derobermasure, 2012; Lapostolle, 2012; Lapostolle, Genelot, 2012). Elles contribuent à professionnaliser, mais seront-elles enseignées de sorte à répondre aux attentes des enseignants en formation aux moments particuliers de leur progression dans le métier? Le seront-elles dans de bonnes conditions?

Il semble que ces sciences humaines et sociales soient en passe de perdre du terrain dans la formation des enseignants à venir. Concernant la formation des enseignants du second degré, elles avaient acquis une certaine légitimité dans les masters, puisqu'elles préparaient à une partie d'épreuve des concours de recrutement. Cette partie consistait en une question portant sur une compétence ainsi dénommée : «Agir en fonctionnaire de l'État... ». Bien que, à la suite d'une certaine contestation émanant des « défenseurs du disciplinaire » dans les épreuves des concours, cette partie n'ait fini par compter que pour un tiers de l'épreuve au lieu d'être une épreuve à part entière, elle avait néanmoins le 
mérite d'exister (Lapostolle, 2012). Or, si cette partie d'épreuve perdure dans les concours du premier degré, elle est désormais supprimée dans ceux du second degré. Cette suppression fait que ces sciences risquent de ne plus trouver de réelle légitimité, ni auprès de ceux qui élaborent les maquettes de formation, ni auprès des étudiants. Elles encourent le risque d'être réduites à la portion congrue dans les maquettes ou de survivre mais dans des conditions où leur enseignement se fera sous forme magistrale, en rupture avec ce qu'il était recommandé de faire il y a quelques vingt années pour qu'elle fasse sens pour les étudiants. Une circulaire de 1992 mentionnait : " Pour assurer la cohésion de la formation et dégager le temps nécessaire au travail personnel, on donnera une place suffisante aux modalités de formation autres que cours, TP, TD : stages, ateliers, travail en petits groupes, tutorat...» (MEN, 1992). Quand il fallait rompre avec des modalités traditionnelles d'enseignement, il était recommandé d'explorer d'autres modalités de formation...

Or, les cours magistraux semblent réapparaitre dans bon nombre d'ESPE sous prétexte d'alignement avec les masters portés par d'autres composantes. Certes, la logique gestionnaire est-elle probablement aux origines de ces choix, mais peut-être ces choix sont-ils aussi la conséquence d'une méconnaissance des processus à l'œuvre dans la formation professionnelle des enseignants...

En somme, ces quelques constats qui ont émergé au lendemain de la «masterisation » et plus récemment, alors que les ESPE se mettent en place, interrogent ce qu'il en est d'un " processus déscolarisation » qui semble être une condition à manipuler avec précaution pour qu'une formation professionnelle digne de ce nom, soit mise en place. Certes, ce terme n'apparaît que rarement en tant que tel dans la littérature scientifique relative à la formation des enseignants, encore moins dans les rapports d'experts - qu'ils se réfèrent ou non à l'international - susceptibles de guider l'action politique. Il témoigne cependant d'une certaine expérience et de retours réflexifs et scientifiques sur cette expérience qui ont émergé au cours des vingt dernières années pendant lesquelles la formation des enseignants n'a cessé d'évoluer (Pôle Nord-Est, 2004, 2013 ; Lapostolle, Grisoni, Solomon, op.cit.). Le recours massif à des enseignants qui ont encore des liens avec le terrain, qui semble être un des piliers de la réforme souhaitée par Vincent Peillon, ministre de l'Education nationale ${ }^{6}$, saura-t-il être le garant de la prise en compte de ce processus ?

En somme, les deux dernières réformes de la formation des enseignants, masterisation et création des ESPE, malgré les nombreuses petites divergences qu'elles présentent, montrent néanmoins un point commun de taille. Tout semble s'être passé, pour chacune d'elles, comme si la volonté de faire converger la formation vers un modèle supranational, dont on ne retenait que les grandes tendances (universitarisation de la formation, meilleur ancrage sur le terrain de la pratique...), tout en prétendant « faire du neuf », avait conduit les décideurs politiques à « faire table rase » de l'expérience acquise dans les IUFM.

\section{Conclusion}

Ces éléments de réflexion à propos du début des ESPE au regard de l'expérience qu'il était possible de tirer du fonctionnement - mais aussi des dysfonctionnements - des IUFM, ne sauraient être montés en généralité, compte tenu du recueil d'information incomplet dont nous disposons quant aux premiers pas des ESPE, au moment où ces lignes sont écrites. Le recueil de données empiriques et l'analyse devront bien entendu être 
développés. D'autres indicateurs pourront aussi être pertinents pour tenter d'évaluer l'efficacité de ces ESPE en matière de professionnalisation des enseignants : la manière dont la recherche en éducation sera réinvestie dans les contenus de formation des masters ; les conditions du déroulement de l'alternance ; les conditions d'une articulation optimale entre formation académique et formation professionnelle, notamment au regard des dispositions dans lesquelles les jeunes enseignants en formations sont pour s'approprier ces contenus... Ces quelques éléments devraient permettre d'évaluer au moins au cours de premières années de fonctionnement la manière dont les ESPE engageront leur mission de professionnalisation des enseignants. Pour autant, il y a lieu de s'interroger à propos de cette création des ESPE.

La volonté affichée par les politiques de rompre avec l'existant est telle, au niveau national, comme au niveau plus local des Universités, qu'il est légitime de se demander par quels canaux ou quels réseaux cette expérience acquise par les IUFM pourra transiter. S'il est vraisemblable que les formateurs des IUFM ont assimilé cette expérience, il convient néanmoins que le contexte institutionnel leur permette de la mettre au profit d'une professionnalisation exigeante. Or les porte-parole de cette expérience, en l'occurrence, les représentants des personnels IUFM ou les directions des IUFM, n'ont semble-t-il pas toujours été en position de faire entendre cette voix dans les instances ou dans les comités de pilotage qui avaient pour mission de préparer la mise place de ces ESPE. Si l'on peut objecter à cette assertion que la situation est évidemment variable d'une université à l'autre, il n'en est pas moins vrai que le cadrage proposé par l'Etat a permis que les situations soient effectivement variables.

Notre propos n'est pas proposer une lecture hagiographique de l'expérience construite par les IUFM, encore moins de nous pencher avec nostalgie sur des instituts qui devaient sans aucun doute évoluer, voire disparaître. Il s'agit simplement d'attirer l'attention sur le fait que la "volonté de faire du neuf ", dont André Legrand (1992) disait déjà, il y quelques vingt années, qu'elle était le point commun de toutes les équipes fraîchement arrivées au pouvoir, que ce soit à un niveau national ou local, ne garantit pas à elle seule la pertinence d'un programme, quand bien même ce programme serait inspiré par un modèle supranational. Si la réforme est nécessaire et si réformer consiste bien souvent à construire contre l'existant, «construire contre cet existant » implique en toute logique que l'on ne l'ignore point ! 


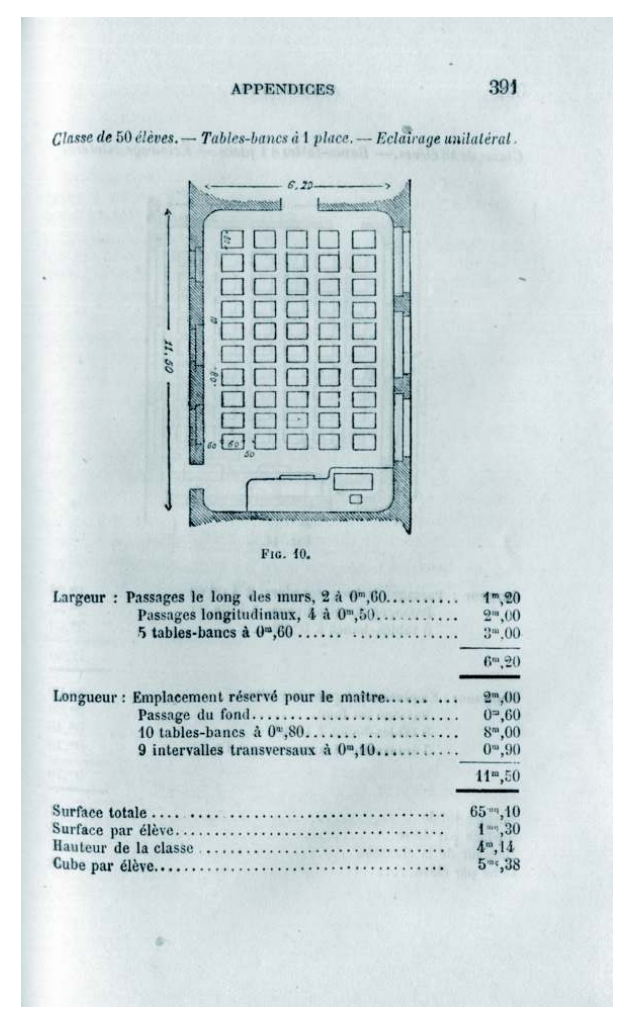

Conférences de pédagogie, manuel des élèves maîtres et des instituteurs. Mariotti, L., Paris : Hachette, $5^{\mathrm{e}}$ édition, 1881. p. 391. Source : Cedrhe 13284.

\section{BIBLIOGRAPHIE}

Abraham, (Yves-Marie), « Du souci scolaire au sérieux managérial, ou comment devenir un HEC », Revue Française de sociologie, 2007/1, VOL. 48, p. 36-66.

Altet (Marguerite), « Les compétences de l'enseignant-professionnel : entre savoirs, schèmes d'action et adaptation, le savoir analyser » in Paquay L., Altet M., Charlier E., Perrenoud P.. Former des enseignants professionnels : quelles stratégies? Quelles compétences? Bruxelles : De Boeck (4e édition), 2011.

Anderson-Levitt (Kathryn), Local Meanings, Global Schooling, Anthropology and World Culture Theory, New York, Palgrave Mac Millan, 2003.

Baillat (Gilles), Niclot (Daniel), Ulma (Dominique), La formation des enseignants en Europe. Approche comparative. Bruxelles, De Boeck, 2010.

Darcos (Xavier), « Propos extraits de l'émission Les grandes gueules », 12 février 2009.

Derobermasure (Antoine), La formation initiale des enseignants et le développement de la réflexivité? Objectivation du concept et analyse des productions orales et écrites des futurs enseignants. Thèse sous la direction de Marc Demeuse, Université de Mons, 2012. 
IGEN, IGAENR, La formation initiale et continue des maîtres. Rapport conjoint. education.gouv.fr, 2003.

Jamet (Laurent), « Une tendance : la formation des enseignants se déporte vers l'école ». La recherche en éducation. Revue de l'association francophone internationale de recherche scientifique en éducation, $\mathrm{N}^{\circ} 9,2013$.

Lapostolle (Guy), Genelot (Sophie), « Du bon usage des sciences humaines et sociales dans la formation des enseignants », Lyon, ENS, Tracés. Revue de sciences humaines, Hors-série, 2012.

Lapostolle (Guy), Chevaillier (Thierry), « Training the teachers in France in the early 2010s' », Journal of education for teaching, vol 37, Issue IV, 2011.

Lapostolle (Guy), Maurel (Laurence), Verney-Carron (Nicole), « Le mémoire professionnel dans la formation initiale des enseignants en France" [The professional report in initial teacher training in France], European Journal of Teacher Education, vol 30, N 4 , ATEE, 2007.

Lapostolle (Guy), Mabilon-Bonfils (Béatrice)., Maurel (Laurence), Agir en fonctionnaire de l'Etat de manière éthique et responsable. Conseils, sujets corrigés, Paris, Armand Colin, 2011.

Lapostolle (Guy) Grisoni (Pascal), Solomon (Rachel), « Le pilotage par l'État de la formation des enseignants du début des années 1990 à nos jours. Les incidences sur le métier de formateur » in Maubant P, Lapostolle G., Gagnon C., La professionnalisation des métiers de l'éducation et de la formation et ses acteurs : sens, fonctions et pratiques du conseil et de l'accompagnement en formation professionnelle, symposium, Congrès de l'AMSE, Reims, 3-8 juin 2012.

Lapostolle (Guy) « La connaissance du système éducatif contribue-t-telle a rendre l'enseignant compétent ? » in Lapostolle (Guy) dir., La formation des enseignants en France. Recherche et Professionnalisation, Éditions universitaires de Dijon, février 2012.

Lapostolle (Guy), Formation initiale des enseignants et professionnalisation : un itinéraire de recherche. Rapport d'Habilitation à diriger des recherches en sciences de l'éducation, soutenu le 3 juillet 2013 à l'Université de Bourgogne.

Mallet (Régis), Brisard (Estelle), « La formation professionnelle des enseignants en France et au Royaume-Uni : dispositifs d'alternance et modèles de formation », Revue française de pédagogie, $\mathrm{N}^{\circ}$ $144,2003$.

Maulini (Olivier), « Nouvelles compétences, anciennes méthodes ? Mouvements et contremouvements dans l'école et la formation des enseignants : le cas de Genève et de la Suisse » Colloque : La formation des enseignants en Europe : approche comparative, 9 et 10 décembre 2008.

MEN, Circulaire $\mathrm{N}^{\circ} 91-202$, Contenus et validation des formations organisées par les IUFM, 2 juillet 1991, JORF, 1991 a.

MEN, Circulaire $\mathrm{N}^{\circ} 91-263$, Modalités de validation de la formation dans les IUFM des professeurs stagiaires lauréats des concours du CAPES, CAPET, CAPEPS, CAPLP2 de l'année 1991-1992 », 30 septembre 1991, JORF, 1991, b.

MEN Circulaire $\mathrm{N}^{\circ}$ 92-118, Préparation des plans de formation des IUFM pour les années universitaires 1992-1994, 20 mars 1992, JORF.

MEN Circulaire $\mathrm{N}^{\circ}$ 2001-150, Accompagnement de l'entrée dans le métier et formation continue des enseignants des premier et second degrés, et des personnels d'éducation et d'orientation, 27 juillet 2001, JORF.

MEN Circulaire $N^{\circ}$ 2002-070, Principes et modalités d'organisation de la deuxième année de formation dans les IUFM », 4 avril 2002, JORF. 
MEN Circulaire $\mathrm{N}^{\circ}$ 2007-045, Mise en œuvre du cahier des charges de la formation des maittres, 23 février 2007, JORF.

MEN « Formation des enseignants : comparaisons internationales. Concertation sur la refondation de l'école de la République ». education.gouv.fr, consulté le 12/08/2013.

MESR Circulaire relative à la mise en place des diplômes nationaux des masters ouverts aux étudiants se destinant aux métiers de l'enseignement, $\mathrm{N}^{\circ}$ 2009-1037 du 23-12-2009, JORF.

Peillon (Vincent), « Les ESPE ne seront pas les IUFM », Le café pédagogique, site consulté le $22 / 8 / 2013$.

Pôle Nord-Est, Être formateur d'enseignants, École d'été du Pôle Nord-Est, 30 juin au 2 juillet, 2004, site CDIUFM, consulté le 23-01-2013.

Pôle Nord-Est, La masterisation, École de printemps du Pôle Nord-Est, 5 avril 2012, Rapport, document interne, IUFM de Franche-Comté, 2012.

Robert (André), Terral (Hervé), Les IUFM et la formation des enseignants aujourd'hui, Paris, PUF, 2000.

Saussez (Frédéric), Allal (Linda), « Réfléchir sur sa pratique : le rôle de l'autoévaluation?» in Mesure et Évaluation en éducation, $\mathrm{N}^{\circ} 30,2007$.

Tardif (Maurice) «Influences internationales et évolutions de la formation des enseignants dans l'espace francophone ». Entretien de Maurice Tardif réalisé par Régis Malet, 2010. Site recherche.formation.org. consulté le 5-12-2012.

Touteduc « Des IUFM au ESPE : les avertissements des inspections générales », paru dans la rubrique Scolaire, le 17 août 2013.

\section{NOTES}

1. Haute École pédagogique de Berne, du Jura et de Neuchâtel.

2. Le cadrage de la formation, en termes de contenus, tel qu'il est proposé au début des années 1990 pour la formation en première et seconde année (MEN, 1991ab ; 1992) disparaît pour céder la place à un cadrage par des compétences (MEN, 2007).

3. La circulaire (MEN, 1991a) mentionnait que la formation devait reposer sur trois piliers : «les stages en établissements scolaires ", « la formation disciplinaire » et " la formation générale et transversale ». Ce dernier pilier invitait sans aucun doute à un recours conséquent aux sciences humaines et sociales : « Connaissance du système éducatif, des publics scolaires... ».

4. «Aujourd'hui (...) les professeurs passent un examen, un concours, ils sont mis dans l'Institut de formation des maîtres, où on leur apprend des théories générales sur l'éducation et puis de temps à autre ils vont remplacer un professeur absent. Ce n'est pas comme ça qu'on forme des gens. Autrement dit, ils sont sans arrêt devant un simulateur de vol. Alors que dans le système que je propose, ils ne seront pas dans un simulateur de vol, ils s'installeront dans le cockpit avec un copilote et ils entreront dans la carrière ».

5. Sur le site touteduc.fr, on peut lire le 21 août 2013 : «L'architecture de la formation qui se met en place" avec les ESPE (écoles supérieures du professorat et de l'éducation) "va demander un haut degré de coopération" entre les universités et l'Éducation nationale, c'est-à-dire l'employeur. Or "l'accompagnement au niveau national de certaines académies pourrait s'avérer nécessaire". En termes diplomatiques, les deux inspections générales mettaient en garde dès le mois de janvier, dans un rapport publié seulement cet été, les ministres de l'Education nationale et de l'Enseignement supérieur, sur les difficultés qu'ils devaient anticiper, s'agissant des relations entre les recteurs, les formateurs et les présidents d'université ». 
6. Vincent Peillon, dans un déjeuner de presse du 30 avril 2013, mentionne que «Les ESPE ne seront pas les IUFM » et envisage d'écarter les enseignants qui se sont trop éloignés du terrain.

\section{RÉSUMÉS}

À partir des années 1980, la formation des enseignants connaîtra dans de nombreux États de profonds bouleversements. La France n'échappera pas à ce mouvement de "professionnalisation » et comme chacun de ces États, elle le "traduira » à sa manière. Cette originalité s'est manifestée par la création des IUFM (Instituts universitaires de formation des maîtres) au début des années 1990. Elle s'est également manifestée, jusqu'à la réforme dite de "masterisation", par le fait de prendre en compte de manière singulière la posture des enseignants en formation, notamment le processus de déscolarisation qui accompagne leur professionnalisation. Or il semble que ni la réforme de la masterisation au tournant des années 2009-2010, ni la création des ESPE (Écoles supérieures du professorat et de l'éducation), à la rentrée 2013, n'ont intégré cette expérience acquise au sein des IUFM. Tout semble s'être passé comme si la volonté de faire converger la formation vers un modèle supra national, dont on ne retenait que les grandes tendances, tout en prétendant "faire du neuf», avait conduit les décideurs politiques à «faire table rase » de l'expérience acquise dans les IUFM.

From the 1980s, teacher training experienced deep changes in many states. France did not escape this trend of "professionalization" and as each of these States, it "translates" it, its own way. This originality is manifested by the creation of IUFMs (University Institutes For Teacher Training) in the early 1990s. It was also apparent, until the reform known as "mastering", by the fact to take into account the singular posture of teachers in training, including the process of outschooling that accompanies their professionalization. But it seems that neither reform mastering, at the turn of the year 2009-2010, nor the creation of ESPE (High Schools of the teaching and education), in September 2013, have incorporated the experience gained in the IUFMs. Everything seems to have happened as if the resolve to bring training to a supra national model, of which are only retained the major trends, while claiming "to make something new" led policymakers not to heed of the experience IUFMs.

\section{INDEX}

Keywords : ESPE, evaluation, mastering, schooling, teacher Training

Mots-clés : déscolarisation, ESPE, évaluation, formation des enseignants, masterisation

\section{AUTEUR}

\section{GUY LAPOSTOLLE}

Maître de conférences HDR en sciences de l'éducation ESPE/IREDU, Université de Bourgogne 